\title{
Language Development during the Fourth Year
}

\section{George \& Julia Brandenburg}

To cite this article: C. George \& Julia Brandenburg (1916) Language Development during the Fourth Year, The Pedagogical Seminary, 23:1, 14-29, DOI: 10.1080/08919402.1916.10534690

To link to this article: http://dx.doi.org/10.1080/08919402.1916.10534690

册 Published online: 30 Aug 2012.

Submit your article to this journal

LII Article views: 4

Q View related articles $\sqsubset$ 


\title{
LANGUAGE DEVELOPMENT DURING THE FOURTH YEAR
}

\author{
By George C. and Julia Brandenburg, Madison, Wisconsin
}

\section{The Vocabulary}

Introduction.-The environment of the child whose vocabulary is discussed in this study was essentially the same during the third and fourth years. The first ten months of the fourth year she lived in Boulder, Colorado, where she had abundant opportunity for outdoor life. Although an only child in the home, there were few days when she did not play somewhat with other children. In general there was more association with other children this year than during the third year. The latter part of her fourth year was spent in Madison, Wisconsin. Here she lived in the central part of the city, where there was scant opportunity to be in the open, although she was often taken for walks to the nearby lakes and parks. On the whole, approximately $80 \%$ of her waking hours during the year has been passed in the company of adults and $20 \%$ in association with other children. ${ }^{1}$ Her favorite playmate in Boulder was a boy nine months younger then she, but she also played with other children varying in age from two to twelve years. $G$ has been in perfect health during the entire fourth year. Her favorite playthings have been three inexpensive dolls; unfortunately she has never had pets for any length of time, but she has always manifested a keen interest in animals of any kind. She is very fond of stories and poems and often entertains herself repeating those she has heard and inventing new ones. In regard to her language development we have always followed a laissez faire policy, as we believe that the best way to secure good linguistic expression is through example rather than precept. Occasionally her language mistakes have been corrected, but more often she has been allowed to correct them of her own accord when she recognized her errors through observation of the speech of other people. Whether as a result of this or not, $G$ has

\footnotetext{
${ }^{1}$ A brief biography of $\mathrm{G}$ for the first three years is given in an article, "The Language of a Three-Year-Old Child," in the Ped. Sem., March, 1915, p. 89 .
} 
always seemed anxious to correct her linguistic errors and to make her speech approximate as nearly as possible that of her adult companions.

Method.-Various methods were employed in obtaining G's vocabulary for the fourth year. Approximately one month following her fourth birthday was spent in observing and recording words used. During the last few days of the period she was tested upon various words which we remembered that she had used previous to this time. Some words not discovered by either of these methods were obtained by using published vocabularies and the dictionary. It was early found that many words which she knew and used at three were no longer in use, and hence were not included in her present vocabulary. Obviously in so limited a period of observation a complete list of words used by the child could not be secured. However, later tests show that comparatively few words have been omitted.

Principles.-Since there is no uniformity of practice as to just what words should be included in a child's vocabulary and for other reasons which will appear later in the discussion, it has seemed best to give the vocabulary both with and without variants. At four years of age $G$ acquires and uses regular inflections without great difficulty, but she evidently does not, for example, gain the use of the past tense and past participle of a verb simply through the acquisition of the present tense. Examples of this are found in the verbs "toot" and "urge" which are used only in the present tense. This is equally true of adjectives and adverbs. Considerable interest centers about the matter of inflections: What part of the vocabulary do variants form at different periods of development? Which forms give the child most trouble? When, if ever, do variants cease to be a source of difficulty? Such questions may be answered only if these forms are recorded in children's vocabularies, at least where the child does not use them without some difficulty.

No words have been included in G's vocabulary which she did not use spontaneously and correctly in conversation. Many numerals, terms from stories, songs, and poems, used in a purely imitative manner have thus been excluded. Names of persons and places used in a similar manner are also not counted.

Size.-Including regular variants except noun plurals, the vocabulary contains 3915 words; excluding all except irregular variants, it contains 3061 words. The vocabulary is 
distributed among the different parts of speech as follows: Nouns 1728, verbs 716 (including variants 1510 ), adjectives 374 (including variants 427 ), adverbs 144 (including variants 150 ), pronouns 42 (including variants 43 ), prepositions 24 , interjections 19, conjunctions 14 . The fact that a normal child of four years may have a speaking vocabulary of almost or fully 4000 words causes one seriously to question the desirability or wisdom of limiting the vocabulary of a spelling book to two or three thousand words. Even though grammatically the variants may not be regarded as separate words, so far as spelling is concerned they certainly must be.

Increase.-The total increase during the year amounts to 1633 words if we count the 580 variants; variants omitted, the increase is only 1053. Since the vocabulary at three years was 2282, variants included, and 2008, variants excluded, the increase is seen to be $71 \%$ in the former case and $52 \%$ in the latter. Children in the public schools build up their vocabularies at the rate of approximately 1400 words per year, exclusive of variants. ${ }^{1}$ Thus we find that G does not acquire words in her fourth year so rapidly as she will later. This is no doubt due to the fact that she gains new words now only through hearing them; whereas later she will get a large per cent of her new words through reading.

The increase in vocabulary is distributed among the parts of speech as follows:

\begin{tabular}{|c|c|c|}
\hline & Words & Per cent \\
\hline 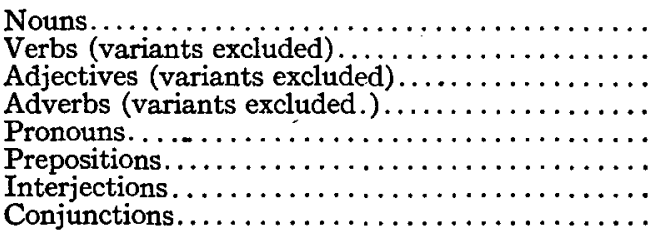 & $\begin{array}{r}557 \\
251 \\
183 \\
46 \\
6 \\
4 \\
4 \\
2\end{array}$ & $\begin{array}{l}47.5 \\
51.8 \\
90.5 \\
46.8 \\
16.9 \\
20.0 \\
26.6 \\
16.6\end{array}$ \\
\hline 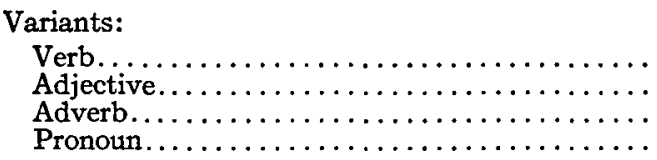 & $\begin{array}{r}527 \\
46 \\
6 \\
1\end{array}$ & \\
\hline Total increase $\ldots \ldots \ldots \ldots \ldots \ldots \ldots \ldots \ldots$ & 1633 & \\
\hline
\end{tabular}

Since the total increase in vocabulary is $52 \%$, it is clear that the nouns have not increased as rapidly as one would expect. The cause of this is probably to be found in the

${ }^{1}$ This fact has been ascertained in an unpublished study which the author made in which the vocabularies of 2000 school children of various grades were tested. 
child's interest and environment during the year and a partial explanation is found in the section dealing with nouns in detail. The greatest per cent of gain is seen to be in adjectives; the increase from 191 at three years to 374 at four years seems to mark this as a period of great growth of interest in and knowledge of the nature and characteristics of the persons, animals, and objects of the child's environment. The increase in verbs and adverbs is apparently about normal. In the other parts of speech few words have been added since $G$ already possessed a sufficient fund of these at the end of the third year to meet her requirements for some time. In fact she had in her vocabulary at that time nearly all the words of these parts of speech which are used in the every-day conversation of her associates.

A very large part of the total increase in words is in variants, the number of these added being 580. At three years of age the variants constituted $12 \%$ of the entire vocabulary; while a year later they amount to $21.8 \%$. The largest per cent of gain in variants during the year is in adjective forms. At three years she had scarcely begun to use comparatives and superlatives, while at four she employs 53 of these forms. It does not satisfy her any longer to say that a new dress is simply "pretty;" she must think of it in relation to other dresses and judge it " prettier " or " prettiest." The first regular adjective variants used, "deeper" and " deepest," were used in connection with playing "make garden;" these and five other regular adjective inflections were used previous to the fourth year. The first adverb forms " slower," " slowest," " faster," " fastest," " longer," and "longest" appeared during the fourth year in connection with play where running was involved. In both cases the necessity of making fine distinctions arose in relation to concrete play situations. Such words as " still," " early," and "naughty,"-static, time, and moral terms-have been used throughout the year, but only in the simple forms. Here vagueness of concepts makes keen discriminations, as yet, impossible; lack of interest may also be a factor in the postponement of the acquisition of such terms.

\section{Comparison of Third and Fourth Year Vocabularies}

An attempt is made in this section to determine to some extent, through a comparison of the contents of the vocabularies at three and four, along what channels mental growth has proceeded during the fourth year. A survey of the following table exhibiting the distribution of the parts of speech in the two vocabularies, shows that the proportions 
have not changed greatly except in the case of adjectives where the increase has been disproportionately large. Naturally in the section of the table in which variants are included

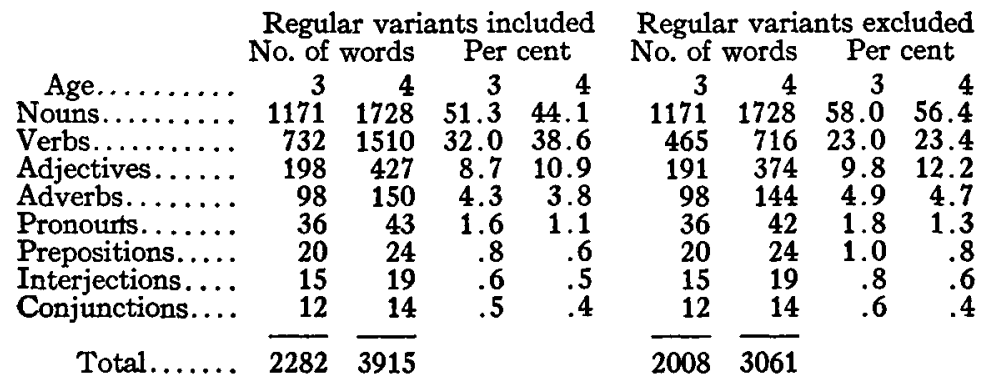

in the calculations, there is seen to be considerable disturbance in the noun and verb elements, due to the large increase in verb inflections. This is significant in revealing the increasing importance of word forms in the vocabulary.

Nouns. - A comparison of the fourth year nouns with those of the previous year suggests some change in the sources of G's vocabulary acquisition. This change is no doubt indicative in some measure of shifting interests and developing mental powers. We have prepared a table to portray as accurately as possible the environmental and interest sources of the nouns. The items are arranged in order of the per cent of increase.

TABLE OF NOUNS

\begin{tabular}{|c|c|c|c|c|c|}
\hline \multicolumn{4}{|c|}{ 年 } & cent & $\begin{array}{l}\text { Per cent } \\
\text { of increas }\end{array}$ \\
\hline Age.................. & $\begin{array}{l}3 \\
8\end{array}$ & 4 & 3 & 4 & 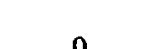 \\
\hline 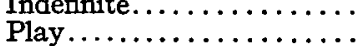 & 176 & $\begin{aligned} 8 \\
196\end{aligned}$ & 14.2 & 11.3 & 17.3 \\
\hline Quantity.....$\ldots \ldots \ldots \ldots$ & 41 & 50 & 3.5 & 2.9 & 21.9 \\
\hline Food...................... & 157 & 193 & 13.4 & 11.1 & 22.9 \\
\hline 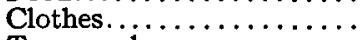 & 69 & 87 & 5.8 & 5.0 & 26.0 \\
\hline Topography............. & 69 & 89 & 5.8 & 5.1 & 28.9 \\
\hline Body................. & 51 & 68 & 4.3 & 3.9 & 33.3 \\
\hline Time..... & 26 & 37 & 2.2 & 2.1 & 42.3 \\
\hline Animals.. & 79 & 117 & 6.7 & 6.7 & 48.4 \\
\hline House... & 187 & 299 & 15.9 & 17.3 & 59.8 \\
\hline Health $\ldots \ldots \ldots \ldots \ldots \ldots$ & 24 & 39 & 2.0 & 2.2 & 62.5 \\
\hline Picture and story..$\ldots \ldots \ldots$ & 9 & 15 & .7 & .8 & 66.6 \\
\hline Civilization $\ldots \ldots \ldots \ldots \ldots$ & 50 & 84 & 4.2 & 4.8 & 68.0 \\
\hline People............... & 124 & 215 & 10.5 & 12.4 & 73.3 \\
\hline$\ldots \ldots \ldots \ldots$ & 6 & & .5 & & 100.0 \\
\hline Abstract. & 83 & 171 & 7.9 & 9.8 & 106.0 \\
\hline 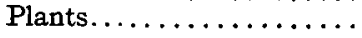 & 21 & & 1.8 & & 128.5 \\
\hline Tote & 1171 & 728 & & & 47.5 \\
\hline
\end{tabular}


At the age of three, we find among the seventeen groups of nouns four relatively large, namely: house, play, food, and people. At four, a fifth group has sprung into prominence and now we find these groups in the following order: house, people, play, food, and abstract. Animals, with 117 terms, are becoming an important interest but still they constitute only $6.7 \%$ of the total nouns at four, just as they did at three. Play and food appear to be relatively less important interests at four than they were at three, while house, people, and abstract terms are relatively more important. The largest per cent of increase is seen to be in plant terms, $128.5 \%$. However, the actual number of words pertaining to plant life is still not large, especially when it is considered that $G$ has spent a large part of her time out of doors. So far she has shown little interest in plant life and the large per cent of increase is to be accounted for partly by the smallness of the three year list and partly by a favoring environment. The large per cents of increase in position, and picture and story terms have little significance since the number of such terms is so small. Picture and story words, as before stated, are generally not included in the vocabulary.

It is not surprising that approximately $40 \%$ of the nouns in use at four years still pertain to objects in the home, play, and food; but it must be noted that the big per cents of growth during the year have not been along these lines. This is not taken to mean that the child is losing interest in these fields, but that she has more or less exhausted them and is beginning to explore new realms. This, no doubt, also explains the relatively small addition to the groups referring to body and clothes. The fact that the greatest expansion has been in abstract terms and in words from out-door sources,--plants, people, and civilization,--suggests a growing interest in, and appreciation of the facts of nature and life apart from herself. The rather slight increase of $28 \%$ in topography terms does not seem to bear out this explanation. We believe, however, that the smallness of this group of nouns at four is due to the fact that when we recorded her vocabulary she was living in Madison, a new environment, and had ceased using, to a great extent, the topography terms of the Boulder environment and had not yet acquired many such terms relating to her new surroundings.

Verbs.-Compared with the verb element in other child vocabularies, this group of words in G's vocabulary is rather large. Excluding regular inflections, we find that at three 
Adverbs. - The following table presents a comparison of the adverbs of the third with those of the fourth year classified according to meaning.

\begin{tabular}{|c|c|c|c|c|c|}
\hline \multirow[b]{2}{*}{ 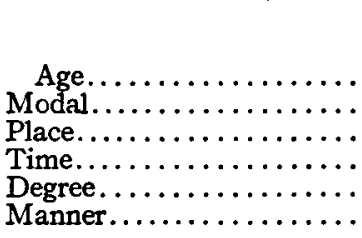 } & \multicolumn{2}{|c|}{ No. of words } & \multicolumn{2}{|c|}{ Per cent } & $\begin{array}{l}\text { Per cent } \\
\text { of increase }\end{array}$ \\
\hline & $\begin{array}{r}3 \\
7 \\
26 \\
28 \\
18 \\
19\end{array}$ & $\begin{array}{r}4 \\
8 \\
34 \\
38 \\
29 \\
35\end{array}$ & $\begin{array}{r}3 \\
7.1 \\
26.5 \\
28.5 \\
18.3 \\
19.4\end{array}$ & $\begin{array}{r}5.4 \\
23.5 \\
26.4 \\
20.1 \\
24.3\end{array}$ & $\begin{array}{l}14.2 \\
30.7 \\
35.7 \\
61.1 \\
84.2\end{array}$ \\
\hline Total................ & 98 & 144 & & & 46.8 \\
\hline
\end{tabular}

Adverbs relating to time and place were the first to appear in G's speech and at three years of age were still the two largest groups, constituting $55 \%$ of the entire number. At four these two groups have fallen to $50 \%$, while those of manner and degree have risen from $37.7 \%$ at three to $44.4 \%$ at four. Time still occupies first place in actual number of words, but adverbs of manner now rank second. The most significant feature of the table is the fact that growth during the year has been largest in adverbs of manner and degree, indicating that interest in action is shifting from the "when and where" aspects to the "how and how much" phases. This is supported by the fact that nouns pertaining to time and place also show relatively small increases.

Conclusion.-Acquisition of new words has proceeded with no apparent interruption during the fourth year; there is nothing to indicate that G's vocabulary growth has not been fully as large as it was in the third year. There has been a significant gain in the fund of variants and in skill in using them, although G's mastery of this phase of language is by no means complete. The use of qualifying terms has become less purely imitative and physical and more spontaneous and meaningful-a change, however, which is still in its infancy. During the year there has been a general shifting of the chief sources of vocabulary augmentation from words relating to simple egoistic interests to those pertaining to people, nature, and abstract ideas.

\section{Vocabulary at Four Years of Age}

A, able, about (adv.), about (prep.), above, abuse (-ed, -ing), accident, ace, ache (n.), ache (-ing), " achoo," acquainted, across (adv.), across (prep.), act (-ed, -ing), afford, afire, afraid, after (adv.), after (prep.), afternoon, afterward, again, against, agent, ago, ahead, ails, ain't, air (n.), air (-ed, -ing), airship. "Alabam," alarm, album, alike, alive, all (n.), all (adv.), allow, all right, almost, alone (a.), alone (adv.), along (adv.), along (prep.), already, always, am, Amen, American, an, and, angel, angry, animal, animal-cracker, ankle, another, answer (-ed), ant, 
anxious, any, anybody, anyhow, anything, anyone, anyway, anywhere, apart, apiece, appetite, apple, apple-sauce, apron, apt, Arapahoe, are, aren't, arm, arm (of chair), around (adv.), around (prep.), arrest, arrow, as (adv.), as (conj.), ashamed, ashes, ashpan, ashpit, ask (-ed, -ing), asleep, aster, at, ate, attic, aunt, auto, automobile, awake, away, awful, awfully, ax.

"Baa," baby (n.), baby (a.), back (n.), back (adv.), back (v.), backward, bacon, bacteria, bad (a.), bad (adv.), bag, bake (-ed, -ing), baked beans, baker, bakerman, baking (n.), balky, ball, banana, band, bang (n.), bang (-ing), bank, barber, barber shop, bare, barefoot, bareheaded, barely, bark (n.), bark (-ing), barn, barrel, basin, basket, basket ball, basting, basting thread, bat (-ing), batch, bath, bathrobe, bathroom, bathtowel, bathtub, batter, bawl, be, beads, "bean," beanbag, beans, bear, beat (-ing), beater, beating ( $\mathrm{n}$.), beautiful, beauty, beaver, because, bed, bedclothes, bedroom, bedtime, bee, beef, beefsteak, been, beer, beerbottle, beet, before, began, beggar, begin (-ing), begun, behave (-ing), behind (adv.), behind (prep.), believe, bell, belly, belong, below, belt, bench, bend (-ing), bent, berry, besides, best, bet, better, between, bib, Bible, bicycle, big (-er, -est), bill, "bing," bird, birthday, biscuit, bit (n.), bit (v.), bite (n.), bite (-ing), bitter, black (a.), black (-ed, -ing), blanket, bleed (-ing), bless, blew, blind, blinds, blister, blocks, blood, bloodberry gum, bloodblister, bloody, bloomers, blot, blotter, blow (-ing), blue, blueing (n.), blueing (v.), bluejay, board, boat, body, boil (-ed, -ing), boiler, bolt, bone, bonnet, book, bookcase, boost, boots, borax, borrow (-ed), " bossy," both (a.), both (pron.), bother (-ed, -ing), bottle, bottom, bought, Boulder, Boulder Canon, Boulder Creek, bound (determined), bounce, bouquet, bow, bowels, bowl, "bow-wow," box, boy, bracelet, braid (-ing), bran-bread, branch, brass, bread, bread-basket, bread-board, bread-can, break (-ing), breakable, breakfast, breakfast-food, breast, breath, breathe (-ing), breeze, brick, brick-cheese, bridge, bright (a.), bright (adv.), Brighton, bring (-ing), broke, broken, broom, broth, brother, brought, brown, brown-bread, brush (n.), brush (-ed, -ing), brute, bubble (n.), bubble (v.), bucket, buckle (n.), buckle (-ed, -ing), bud, bug, "bugger," buggy, build (-ing), building (n.), built, bulldog, bullet, " bum," bumblebee, bump (n.), bump (-ed), bunch, bunny, bureau, burglar, burn (n.), burn (-ing), burnt, burro, bush, bushel, business, " bust," busy, but, butcher, butcher-knife, butter, butterfly, buttermilk, button (n.), button (-ed, -ing), buttonhole, buy (-ing), buzz (-ing), by, by-and-by, bye-bye.

Cabbage, cage, cake, calf, calico, call (-ed, -ing), came, camel, Camera, camphor, campus, can (n.), can (v.), candle, candlestick, candy, cane, cannon, can't, cantaloupe, cap, car, card, cardboard, care (n.), care (v.), careful, careless, carpet, carrot, carry (-ed, -ing), cart, cat, catalogue, catastrophe, catch (-ing), caterpillar, Catholic, caught, ceiling, celery, cellar, cement, cents, certainly, chafing-dish, chain, chair, " chair (-ing)," chamber, change ( $\mathrm{n}$. ), change (v.), chase (-ed, -ing) checker, checkerboard, cheek, cheese, chew (-ed, -ing), chicken, chicken-feed, chickenhouse, chick-feed, child, children, chilly, chimney, chin, chip, chocolate, choke (-ing), " choo-choo," chop (-ed, -ing), chopper, Christmas, Christmas-tree, "chuc-a-doo," church, cigar, cinnamon, cinnamon-roll, circus, cistern, city, clap (-ed, -ing), class, clean (a.), clean (-ed, -ing), clear, climb (-ed, -ing), clip, clippers, clock, close (a.), close (-ed, -ing), closet, clothes, clothes-bag, clothes-basket, clothes-brush, clothes-line, clothespin, clothes-stick, cloud, cloudy, clown, club, " cluck," coal, coal-bucket, coal-man, coal-scuttle, coal-shovel, coarse, coat, coax, "cock-a-doodledoo," cocoa, cocoanut, codfish, coffee, coffee-grounds, coffee-pot, cold (a.), cold (n.), cold-cream, collar, color, Colorado, colored (a.), comb (n.) comb (-ed, -ing), come (-ing), comfortable, company, condensed-milk conductor, Congregational, cook (n.), cook (-ed, -ing), cook-book, cookies 
cool, copy (n.), copy (v.), cord, core, cork, corn, corn-bread, corner, cornflakes, cornmeal, correct, corset, cost, cotton, couch, cough (n.), cough (-ed, ing), could, couldn't, count, country, couple, cousin, cover (n.), cover (-ed, -ing), cow, coyote, crack (n.), crack (-ed, -ing), cracker, cradle, cranberries, crank (n.), crank (-ed, -ing), cranky, crawl (-ed, -ing), crazy, cream, cream-pitcher, creek, creep (-ing), crept, crib, cribbage, cribbage, board, crochet, crochet-hook, cross (a.), cross (-ed, -ing), crown, crumb, crust, cry (-ed, -ing), cry-baby, cuddle, cuff, cuff-button, cunning (-est), cup, cupboard, cupful, curl (n.), curl (-ed, -ing), curly, currant, curtain, cushion, custard, cut (-ing), cute.

Dab, dabble (-ing), Daddy, dainty, damp, damper, dance (n.), dance (-ed, -ing), dancer, dandelion, dandy, danger, dangerous, dare, dark, darkness, darling, darn (-ing), darning-needle, dashboard, date, daub, daughter, day, daylight, daytime, dead, deal (n.), deal (-ing), dear (n.), dear (a.), dearie, death, December, decent, decide (-ed), deck, decorate, decoration, deep (-er, -est), delicious, delightful, Denver, Denver Post, depot, deserve, desk, dessert, destroy (-ing), devil, dew, diamond, dictionary, did, didn't, die (-ed, -ing), difference, different, dig (-ing), dimple, ding-dong, " dingy," dining-car, dining-room, dinner, dip (-ed, -ing), dipper, direction, dirty, disappear (-ed), dish, dish-cloth, dish-pan, disturb (-ing), ditch, dizzy, do (-ing), doctor (n.), doctor (-ing), does, doesn't, dog, doggie, doily, doll, dollar, doll-buggy, dollie, done, donkey, don't, door, door-knob, dose, dot, double, dough, doughnuts, doughy, down, downhill, downstairs, dozen, draft, drain, drank, draw (-ing), drawer, drawers, dreadful, dreadfully, dream (n.), dream (-ed, -ing), dreamt, dress (n.), dress (-ed, -ing), dresser, dressmaker, drew, dribble (-ed, -ing), drink (n.), drink (-ing), drip (-ed, -ing), drive (-ing), driver, drizzly, drop (n.), drop (-ed, -ing), drove, drown (-ed), drugstore, drum, drumstick, drunk, dry (a.), dry (-ed, -ing), duck, dug, dull, dump, dust (n.), dust (-ed, -ing), dustcloth, dustpan, dusty, Dutch, Dutchman.

Each (a.), each (pron.), ear, early, East, Easter, Easter-egg, easy, eat (-ing), eaten, eatables, edge, " ee-ee," egg, eggbeater, eight, either, elbow, electric, electricity, electric-light, elephant, eleven, else (pron.), else (adv.), embroider (-ed, -ing), empty (a.), empty (-ed, -ing), end, endways, engine, engineer, enjoy, enough (n.), enough (a.), entirely, envelope, errand, escape, even (a.), even (adv.), evening, ever, evergreen, evermore, every, everybody, everyday, everyone, everything, everywhere, exactly, excellent, except, excited, excuse, exercise (-ing), expect (-ing), extra, eye, eyebrow, eyelash, eyelid, eye-winker.

Face, face-cream, fact, fade, fair, fairy, Fairy Soap, fall (n.), fall (-ing), family, fan (n.), fan (-ed, -ing), fancy, far, farm, farther, fashion, fashionable, fast (a.) (-er, -est), fast (adv.) (-er, -est), fasten (-ed, -ing), fastener, fat (-er, -est), father, faucet, fault, fear, fearful, fearfully, feast, feather, feather-bed, February, fed, feed (n.), feed (-ing), feel (-ing) (trans.), feel (-ing) (intrans.), feet, fell, fellow, felt, fence, fever, feverish, few, fib (n.), fib (-ed, -ing), fiddle, fiddle-sticks, field, fierce, fifteen, fifty, fig, fight (-ing), fight (n.), fill (-ed, -ing), film, finally, find (-ing), fine (-er, -est), finger, finger-nail, finish (-ed, -ing), fire, fireplace, first, fish (n.), fish (-ed, -ing), fist, fit (a.), fit (v.), five, fix (n)., fix (-ed, -ing), flag, flapping, flat, flat-iron, flew, floor, flour, flower, fly (n.), fly (-ing), fold (-ed, -ing), folks, follow (-ed, -ing), food, fool (-ed, -ing), foolish, foot, foot (of bed), football, footstool, for, forehead, forenoon, forever, forget, forgot, forgotten, fork, forty, found, four, Fourth of July, fresh, freeze (-ing), freight, Friday, friend, fright, fringe, frisky, frog, front, frontdoor, frost (n.), frost (-ing), frosting (n.), frosty, froze, frozen, fruit, fry (-ed, -ing), frying-pan, fudge, fuel, full (-er, -est), fun, funeral, funny (-er, -est), " funnies," fur, furniture, fuss (n.), fuss (-ed, -ing), fussy, fuzzy.

Game, garden, garter, " garter" (-ing, -ed), gas, gasoline, gate, gather (-ed, -ing), gelatine, gems, gentle, gentleman, German, get (-ing), " get- 
up," giggle (-ed, -ing), giggler, girl, give (-ing), glad, glass, glasses (spectacles), glimpse, gloomy, gloves, glue (n.), glue (-ed, -ing), go (-ing), " gobble," gobble (-ed, -ing), God, gold, gone, good, good-bye, goodmorning, good-night, goody, goose, got, grab (-ed, -ing), gracious, graham, grand, grandfather, Grandma, grandmother, Grandpa, grape, grass, grasshopper, grate, grater, gravy, gray, grease (n.), grease (-ed, -ing), greasy, great, green, griddle, grin (-ing), groceries, grocery-man, grocerystore, ground, grow (-ed, -ing), grunt (-ed, -ing), guess, guinea pig, gum, gun, gunny sack.

Ha ha, had, hadn't, hail (n.), hail (-ing), hair, hair-brush, hair-pin, hairy, half, hall, Hallow e'en, ham, hammer (n.), hammer (-ed, -ing), hand (n.), hand (-ed), handful, handkerchief, handle (n.), handle (-ing), handy, hang (-ing), happen, happy, hard (a.), hard (adv.), hardly, harm, harness (n.), harness (-ed, -ing), has, hasn't, hat, hate, hatchet, hatpin, haul (-ing), have haven't, hay, hazelnut, he, head, headache, hear, heard, hearse, heart, heat (n.), heat (-ing), heaven, heavy, hedge, heel, held, hello, " helloed," held (-ed, -ing), hen, her (-s), here, herself, hey, hickory nuts, hid, hide (-ing), high (-er, -est), highchair, hill, hilly, him, himself, hinge, hippety-hop (n.), hippety-hop (-ed, -ing), hit (-ing), hitch-up (-ed, -ing), hoe (n.), hoe (-ed, -ing), hog, hold (-ing), hole, holler (-ed, -ing), home, honest, honey, honeybee, honeysuckle, hood, hook (n.), hook (-ed, -ing), hope (-ing), horn, horrible, horse, horseback, horseradish, horseshoe, horsie, hose, hospital, hot, hotwater-bottle, hound, house, how, "how de do," howl (-ed, -ing), hug (n.), hug (-ed, -ing), hundred, hung, hungry, hunt (-ed, -ing), hurry (-ed, -ing), hurt (-ing), " hurted," husband, hush, hustle (n.), hustle (-ed, -ing).

I, ice, icecream, icicle, icy, idea, if, imagine, in (adv.), in (prep.), incubator, indeed, Indian, ink, inkbottle, inky, inside, instead, intend, interurban, into, Iowa, Irish potato, iron (n.), iron (-ed, -ing), ironing (n.), ironing-board, iron-holder, is, isn't, it, itches, its, itself.

Jabber (-ed, -ing), jacket, Jack Frost, jack o'lantern, jail, jam, January, jar (n.), jar (-ed, -ing), jaw, jelly, jellyroll, jerk, (n.), jerk (-ed, -ing), jiggle (-ed, -ing), jingle (-ed, -ing), job, Johnson St., joke (n.), joke (-ing), jug, juice, juicy, July, jump (n.), jump (-ed, -ing), jumper, junk, just.

Keep (-ing), kept, kernel, kettle, key, keyhole, Kewpie, kick (n.), kick (-ed, -ing), kids, kill (-ed, -ing), kimona, kind (n.), kind (a.), kindle (-ed, -ing), kindling (n.), kiss (n.), kiss (-ed, -ing), kitchen, kitten, kitty, kitty-hood, knead (-ing), knee, kneel (-ing), knew, knife, knock (n.), knock (-ed, -ing), knot (n.), knot (-ed, -ing), know (-ed, -ing), knowledge, kodak.

Lace (n)., lace (-ed, -ing), lack, ladder, lady, lake, lamb, lame, lamp, land, " land sakes," lantern, lap, lapful, lard, large (-er, -est), last (adv.), last (a.), last (-ed, -ing), latch (n.), latch (-ed, -ing), late (-er, -est), lately, lather, laugh (n.), laugh (-ed, -ing), laundry-bag, lawn, lay (-ed, -ing), lavatory, lazy, lead (-ing), lead-pencil, leaf, leak (n.), leak (-ed, -ing), leaky, lean (a.), lean (-ed, -ing), learn (-ed, -ing), leather, leave (-ing), leaves, led, left (a.), left (v.), leg, leggings, lemon, lemonade, lend, lesson, let (-ing), let's, letter, letters (of alphabet), lettuce, level, liable, library, lick (-ed, -ing), lid, lie (-ing), lie (n.), lift (-ed, -ing), light (n.), light (-ing) light (a.) (weight), light (a.) (color), like (n.), like (a.), like (adv.), like (-ed), likely, lilac, limb, lily, limber, line, lining, lion, lip, list, listen (-ed, -ing), lit, litter (n.), litter (-ed), little (n.), little (a.), little (adv.), lively, live (-ed, ing), load (n.), load (-ed, -ing), loaf, loaves, lock (-ed, -ing), locket, London, lonesome, long (a.) (-er, -est), long (adv.) (-er, -est), look (-ed, -ing), looking-glass, loose (a.), loose (v.), lose (-ing), lost (v.), lost (a.), lot, loud (-er, est), lounge, love (-ing), lovely, low (a.), low (adv.), luck, lucky, lump, lunch.

Macaroni, machine, machinery, mad, made, Madison, magazine, mail 
(n.), mail (-ed, -ing), mailbox, mail-carrier, mail-man, make (-ing), Mamma, man, manage, manners, many (n.), many (a.), maple-sugar, marble, March, march (-ing), mark (n.), mark (-ed, -ing), marmalade, marshmallow, mash (-ed, -ing), match, matchbox, matter, mattress, may, maybe, me, meal, mean (a.), mean (v.), meant, measles, measure (n.), measure (-ed, -ing), meat, meat-market, meddle (-ing), medicine, meet, melon, melt (-ed, -ing), mend (-ed, -ing), mention, " meow," mercy, merry-go-round, mess, met, Methodist, mice, middle, might (n.), might (v.), mighty, mile, milk (n.), milk (-ed, -ing), milk-bottle, milk-man, milk-ticket, mill, mince-pie, mind (n.), mind (ed), " mind-you," mine, minute, mischief, Miss, mistake, mistaken, misunderstand, misunderstood, mitten, mix (-ed, -ing), mix-up, molasses, moment, Monday, money, monkey, monster, "moo," moon, mop (n.), mop (-ed, -ing), more (n.), more (a.), more (adv.), morning, most (n.), most (a.), mosquito, Mother, mother-hen, motor-car, motor-cycle, mountain, mouse, mouse-trap. mouth, mouthful, move (-ed, -ing), Mr., Mrs., Mr. Cold, Mr. Splatter, Mr. Warm, Mr. Wind, mucilage, mud, muddle, muddy, muff, muffin, mule, mush, music, muss, muss-up, must, mustn't, my, myself.

"Na," nail (n.), nail (-ed, -ing), name, nap (n.), napping (v.), napkin, narrow, nasty, naughty, near (adv.), near (prep.), nearly, neck, necklace, necktie, need, needle, (n.) " needle " (v.), needn't, negro, neighbor, neighborhood, neither, nervous, nest, never, new (-er, -est), news, newsboy, newspaper, next, nice, nickel, night, night-dress, night-gown, " nightie," nine, no, nobody, noise, noisy, No Mam, none, nonsense, noon, nor, North, nose, No Sir, not, note, notebook, nothing, notice (-ed), notion, now, nozzle, nuisance, number, nut, nut-cracker, nut-pick, nutshell.

Oatmeal, occasionally, o'clock, October, of, off, office, often, $\mathrm{Oh}$, "Oh land," "Oh sugar," old (-er, -est), oil, oil (-ed, -ing), oil-can, oil-cloth, olive, olive oil, on (adv.), on (prep.), once, one (n.), one (a.), onion, onion sets, only, open (a.), open (-ed, -ing), or, orange, orange-juice, orchard, order, organ, ornament, other, "ouch," ought, oughtn't, our, ours, ourselves, out, outdoors, oven, over (adv.), over (prep.), overalls, overcoat, own, "ow," oysters.

Pack (-ed, -ing), package, paddie, paddle (-ed, -ing), paddling (n.), pail, pailful, pain, paint (n.), paint (-ed, -ing), paint-brush, pair, pan, pancake, pansy, panties, pantry, pants, Papa, paper (n.), paper (-ed, -ing), paper-boy, paper-dolls, parade, paragraph, parasol, Parcel Post, pardon, pare (-ed, -ing), park, parlor, parrot, part (n.), part (v.), particular, partly, party, pass (-ed, -ing), past (adv.), past (prep.), paste (n.), paste (-ed, -ing), pasteboard, pasture, pat (n.), pat (-ed, -ing), patch (n.), patch (-ed, -ing), path, patter, pattern, patty-cake, pay (-ed, -ing), pavement, peach, Pearl St., peanut, peas, peculiar, peddle, peddler, peek (-ed, -ing), peek-a-boo, peel (-ed, -ing), peeling (n.), peep (-ed, -ing), peep-peep, pen, pensil, penny, people, pepper, peppermint, perfect, perform (-ed, -ing), performance, person, pester (-ed, -ing), pet (n.), pet (-ed, -ing), petticoat, phenolax, physic, piano, piano stool, pick (-ed, -ing), pickle, picnic, picture, picture-book, picture-hook, picture-show, pie, piece, pie-crust, pieplant, pig, pigeon, piggish, pigpen, pile (n.), pile (-ed, -ing), pillow, pillowcase, pimple, pin (n.), pin (-ed, -ing), pinch (n.), pinch (-ed, -ing), pincushion, pink, pipe, pitcher, pity, place, plain (a.), plain (adv.), plan, plane, plant (n.), plant (-ed, -ing), plaster, plate, plateful, platter, play (n.), play (a.), play (-ed, -ing), play (-ed, ing-) (the piano), playhouse, playmate, plaything, pleasant, please, plenty, plum, poached eggs, pocket, pocketbook, pod, point (n.), point (-ed, -ing), poison, poke (-ed, -ing), poker, pole, policeman, pony, poodle dog, poor, pop (-ed, -ing), popcorn, porch, pork-chops, post, postage stamps, post office, postal card, pot, potato, potato masher, pound (n.), pound (-ed, -ing), pounder, pour (-ed, -ing), pout (-ed, -ing), powder (n.), powder 
(-ed, -ing), powerful, powerfully, practice (-ing), pray (-ed, -ing), prayer prayer book, "prayers" (v.), preach (-ed, -ing), preacher, precious, present (for the), present (gift), preserves, press (-ed, -ing), pressing (n.), presume, pretend (-ed, -ing), pretty (a.), pretty (adv.), price, prick (-ed, -ing), prickly, priest, prince, proceed, proceeding (n.), promise (n.), promise (-ed, -ing), prop (-ed, -ing), proper, prunes, Pshaw, pudding, puddle, puff (n.), puff (-ed, -ing), pull (-ed, -ing), pump (n.), pump (-ed, -ing), pumpkin, punch (n.), punch (-ed, -ing), punish (-ed, -ing), pup, puppy, pure, purpose, purr (-ed, -ing), purse, push (n.), push (-ed, -ing), pussy, pussy willow, put (-ing), putty (n.), putty (-ing).

"Quack," quarrel (n.), quarrel (-ed, -ing), queen, queer, question, quick (-er, -est), quiet, quilt, quit, quite.

Rabbit, race, racket, radish, rag, ragged, rail, railroad, rain (n.), rain (-ed, -ing), rainbow, raindrop, rainy, raise (-ed, -ing), raisin, rake (n.), rake (-ed, -ing), ran, rang, range, rap (-ed, -ing), rascal, raspberry, rat, rather, rattle (n.), rattle (-ed, -ing), ravelling, raw, razor, razor-strop, reach (-ed, -ing), read (-ing), read (past tense), reader, reading (n.), ready, real, realize, really (a.), really (adv.), reason, reckon, red, refrigerator, regular, remember (-ed, -ing), rent (n.), rent (v.), reservoir, rest (n.) (take a rest), rest (remainder), rest (-ed, -ing), restaurant, rheumatism, rhubarb, rib, ribbon, rice, rice pudding, rich, rid, ride (n.), ride (-ing), ridiculous, right (a.), right (adv.), " rightwards," ring (n.), ring (-ing), rinse (-ed, -ing), rip (-ed, -ing), ripe, risk, river, road, roar (-ed, -ing), roast (-ed, -ing), roast-beef, rob (-ed, -ing), robber, robe, robin, rock (n.), rock (-ed, -ing), rock-a-bye, rocking-chair, rod, rode, roll (n.), roll (-ed, -ing), rolling-pin, roly-poly, Roman-meal, romp (-ed, -ing), rompers, roof, room, roost, rooster, root, rope, rose, rosebush, rosy, rotten, rough (a.), rough (adv.), round (n.), round (a.), roustabout, row, row, rub (-ed, -ing), rubber, rubbers, rubbish, rubbish-pile, ruffe, rug, ruin (-ed, -ing), ruler, run (n.), run (-ing), runaway (n.), runaway (a.), rung, runner, runt, rush (n.), rush (-ed, -ing), rust, rustle, rusty.

Sack, sackful, sad, saddle, safe, safetypin, sage, said, sail (-ed, -ing), sailboat, saleratus, salmon, salt (n.), salt (-ed, -ing), salts, salt-cellar, salty, salve, Salvation Army, same, sample, sand, sandman, sandwich, sandy, sandy-andy, Santa Claus, sash, sat, satchel, satin, satisfy (-ed, -ing), satisfactory, Saturday, sauce, saucer, saucy, sauer kraut, sausage, save (-ed, -ing), saw (n.), saw (-ed, -ing), saw (past intrans.), sawdust, say (-ing), scalawag, scab, scales, scalloped, scar, scarce (n.), scare (-ed, -ing), scarce, scarcely, scarf, scheme, schemer, school, school-house, schoolteacher, scissors, scold (-ed, -ing), scoop (-ed, -ing), scorch (-ed, -ing), scowl (-ed, -ing), scrap, scrap-book, scrape (-ed, -ing), scratch (n.), scratch (-ed, -ing), scream (-ed, -ing), screech (-ed, -ing), screen, screendoor, screw (n.), screw (-ed, -ing), screw-driver, scrub (n.), scrub (-ed, -ing), scrubbrush, scrumptious, seam, seat (n.), seat (v.), second (a.), second (n.), secret, secretary, see, see (understand), seed, seek, seem (-ed), seesaw seldom, selfish, sell (-ing), send (-ing), senna, sense, sensible, sent, separate (a.), separate (-ed, -ing), September, sermon, served, set (n.), set (v.), setting hen, settle (-ed, -ing), seven, several, sew (-ed, -ing), sewer (one sewing), sewer (drain), sewer-pipe, sewing (n.), sewing-machine, shade, shadow, shady, shake (n.), shake (-ing), shaking (n.), shaky, shall, shame, shampoo (n.), shampoo (-ed, -ing), shape, share, sharp, sharpen (-ed, ing), shave (-ed, -ing), shaving-brush, shawl, she, shears, shed, sheep, sheet, shelf, shell (n.), shell (-ed, -ing), shelves, shine (-ing), shingle, shiny, shirt, shoe, shoe-blacking, shoe-box, shoe-string, shook, shoot (-ing), shooter, shop (-ing), short (-er, -est), shot, should, shoulder, shoulder-roast, shouldn't, shove (n.), shove (-ed, -ing), shovel (n.), shovel (-ed, -ing), show (n.), show (-ed, -ing), shower, showery, " shucks," shuffle, shut (a.), shut (-ing), sick, sickly, side, sidewalk, sideways, sift (-ed, -ing), 
sifter, sigh, sight, sign, silk, silky, silly, simply, silver, silver polish, silverware, since, sing (-ing), singing (n.), single, sink, sinner, sip (-ed, -ing), sir, sister, Sister (Catholic), sit (-ing), six, size, "skedaddle," "skidoo," skim, skin, skinny, skip (-ed, -ing), skirt, sky, slam (n.), slam (-ed, -ing), slap (n.), slap (-ed, -ing), sled, sleep (n.), sleep (-ing), sleeper, sleepy, sleepy-head, sleeve, sleigh-ride, sleigh-riding, slept, slice, slick, slid, slide (-ing), slingshot, slip (-ed, -ing), slipper, slippery, sliver, slobber (-ed, -ing), slop (-ed, -ing), sloppy, slow (a.), (-er, -est) slow (adv.), (-er, -est) smack (n.), smack (-ed, -ing), small (-er, -est), smart (a.), smart (-ed, -ing), smarty, smash (-ed, -ing), smell (n.), smell (-ed, -ing), smeller, smile (n.), smile (-ed, -ing), smoke (n.), smoke (-ed, -ing), smoky, smooth (-er, -est), snap (n.), snap (-ed, -ing), snarl, snatch (-ed, -ing), sneeze (n.), sneeze (-ed, -ing), snooze (n.), snooze (-ing), snoozer, snore (-ed, -ing), snow (n.), snow (-ed, -ing), snowball (n.), snowball (-ing), snowbank, snowdrift, snowman, snowy, snuff (-ed, -ing), so (adv.), so (conj.), soak (-ed, -ing), soap (n.), soap (-ed, -ing), soapdish, soapy, sob (-ed, -ing), sock, soda, sodawater, soft (-er, -est), soil (-ed, -ing), sold, sole, solid, somebody, somersault, something, sometimes, someway, somewhere, son, song, songbook, soon, soot, sooty, sore, (n.), sore (a.), sorry, sorts, sound (n.), sound (-ed), soup, soupbone, South, sour, sour milk, space, spank, (n.) spank (-ed, -ing), spare, sparkle (-ed, -ing), sparks, sparrow, spat, speak, spear (n.), spear (-ed, -ing), speck, spell (-ed, -ing), spend, spider, spiderweb, spill (-ed, -ing), spilt, spit (n.), spit (-ing), spite, splash (-ed, -ing), splasher, splatter (n.), splatter (-ing), splendid, split (-ing), spoil (-ed, -ing), spool, spoon, spoonful, sport, spot, spotted, spread (n.), spread (-ing), spring, sprinkle (-ed, -ing), sprinkler, sprint (-ed, -ing), spry, squall (-ed, -ing), square, squash, squawk (-ed, -ing), squawker, squeak, squeal (-ed, -ing), squeeze (n.), squeeze (-ed, -ing), squib (-ed, -ing), squint (-ing), squirrel, stack, stain (n.), stain (-ed, -ing), stairs, stalk, stamp, stand (-ing), star, starch (n.), starch (-ed, -ing), stare (-ed, -ing), start (n.), start (-ed, -ing), starve (-ed, -ing), state (of affairs), station, stay (n.), stay (-ed, -ing), steady, steak, steal (-ing), steam, steaming, steam-cars, steep, steeple, stem, step (n.), step (-ed, -ing), stew, stick (n.), stick (-ing), stick candy, sticker, stickpin, sticky, stiff, stile, still (a.), still (adv.), sting, stinger, stir (-ed, -ing), stitch (n.), stitch (-ed, -ing), stocking, stocking-cap, stole, stomach, stone, stood, stool, stoop (-ed, -ing), stop (-ed, -ing), store, storeroom, storm, stormy, story, storybook, stove, stovepipe, straight (a.), straight (adv.), straighten (-ed, -ing), strain (-ed, -ing), strainer, strange, strap, straw, strawberry, stream, street, streetcar, stretch (-ed, -ing), strike, striking, string, strip (n.), strip (-ed, -ing), stripe, striped, strong, stuck, study (n.), study (-ed, -ing), studying (n.), stuff, stumble (-ed), stung, stunt, style, stylish, susbtantial, such, suck (-ed, -ing), sucker, sudden, suds, suffer, sufficient, sugar, sugarbowl, sugarcan, suit (n.), suit (v.), suitcase, sulphur, sultry, summer, sun, sunburned, Sunday, Sunday School, sung, sunlight, sunny, sunshine, sunshiny, supper, supporters, suppose (-ed, -ing), sure, surely, surprise (n.), surprise (-ed, -ing), suspenders, swallow (-ed, -ing), sweat (-ing), sweater, sweaty, sweep (-ing), sweeper, sweet, sweet potato, sweet peas, sweetheart, swell (-ed, -ing), swept, swift, "swipe," swing (n.), swing (-ing), swing-board, swing-rope, switch (n.), switch (-ed, -ing), syrup.

Tabby, table, tablecloth, tablespoon, tack, tackle (-ed, -ing), tail, take (ing), taken, tale, talk (n.), talk (-ed, -ing), tall (-er, -est), tame, tan, tangle (n.), tangle (-ed), tapioca, tar-soap, tart, task, tassel, taste (n.), taste (-ed, -ing), taught, tea, tea-ball, teacup, teach, teacher, teakettle, tea-party, teapot, tear (n.), tear (-ing), tears, tease (n.), tease (-ed, -ing), teaspoon, Teddy-bear, "teensy-weensy," teeter-totter, teeth, telephone (n.), telephone (-ed, -ing), telescope, tell (-ing), temper, ten, tend (-ed, 
-ing), tennis, teninis racket, tent, terrible, terribly, than, thank (-ed), thankful, Thanksgiving, that (a.), that (pron.), thawing, the, theatre, them, then, there, thermometer, these (a.), these (pron.), thesis, they, thick (-er), thimble, thin (-ed, -ing), things, think (-ing), third, thirsty, thirteen, thirty, this (a.), this (pron.), those, though, thought, thread (n.), thread (-ing), thread picker, three, threw, throat, through (adv.), through (prep.), throw (-ing), thrower, throw-up, thumb, thunder (n.), thunder (-ed, -ing), Thursday, ticket, ticking, tickle (-ed, -ing), ticktock, tie (n.), tie (-ed, -ing), tiger, tight, tighten, tights, till, time, tin, tincup, ting-a-ling, tiny, tip (n.), tip (-ed, -ing), tiptoe, tire, tired, to, toad, toast (n.), toast (-ed, -ing), today, toddle (-ed, -ing), toe, toenail, together, toilet, told, tomato, tomboy, tom-cat, tomorrow, tongue, tonic, tonight, too, took, tools, toot, tooth, toothbrush, toothpick, "too too car," top, tore, torment (-ed, -ing), torn, toss (-ed, -ing), touch (n.), touch (-ed, -ing), tough, towards, towel, town, toy, track, trade (n.), trade (-ed, -ing), train (n.), train (-ed, -ing), tramp, trap, trash, treat (n.), treat (-ed, -ing), tree, trick, tricycle, trifle, trim, trot (-ed, -ing), trotting harness, trouble, truck, true, truly, trunk, trunk (of elephant), truth, try (-ed, -ing), tub, tuck, (-ed, -ing), Tuesday, tumble (n.), tumble (-ed, -ing), tumbler, tune, turkey, "Turkey Day," turn (-ed, -ing), turns, tussle, "tweet," twelve, twenty, twenty-fourth, twice, twin, twist (-ed, -ing), two, typewrite (-ing), typewriter.

Ugly, umbrella, unable, unbutton (-ed, -ing), uncle, Uncle Sam, “ unclose," uncover (ed, ing-), under (adv.), under (prep.), underclothes, underneath, underside, underskirt, understand, underwear, undo (-ing), undone, undress (-ed, -ing), " unhanged," unhappy, unhitch (-ed, -ing), unionsuit, University, unkind, unlace (-ed, -ing), unlatch (-ed, -ing), unless, unload (-ed, -ing), unlock (-ed, -ing), unloose (-ed, -ing), unpin (-ed, -ing), untangle (-ed, -ing), untie (-ed, -ing), until, unwind (-ing), unwound, up (adv.), up (prep.), uphill, upper, upside down, upstairs, urge, use (n.), use (-ed, -ing), used to, useless, usually.

Vaccinate (-ed), valentine, variety, varnish (n.), varnish (-ed, -ing), vase, vaseline, vegetable, veil, velvet, verse, very, vessel, vest, victuals, vine, vinegar, visit (n.), visit (-ed, -ing), visitor, voice, vomit (-ed, -ing).

Wabbly, wad (n.), wad (-ed, -ing), wade (-ed, -ing), wafers, wag (-ed, -ing), wait (n.), wait (-ed, -ing), wake (-ed, -ing), "waked-up," walk (n.), walk (-ed, -ing), walker, wall, wallpaper, walnut, want (-ed, -ing), war, warm (-er, -est), warm (-ed, -ing), wart, was, wash (-ed, -ing), washbasin, washboard, washing (n.), washrag, wasn't, wasp, waste (n.), waste (-ed, -ing), watch (n.), watch (-ed, -ing), water (n.), water (-ed, -ing), watermelon, water-pitcher, wave (-ed, -ing), wax, way, we, wear (-ing), weary, weather, Wednesday, "wee-wee," weed (n.), weed (-ed, -ing), weedy, week, weeping, weigh (-ed, -ing), welcome, well (n.), well (a.), well (adv.), well (interj.), went, were, wet (a.), wet (-ed, -ing), whack (n.), whack (-ed), what, whatever, wheat, "whee," wheel (n.), wheel (-ed, -ing), wheelbarrow, when, whenever, whereabouts, where, wherever, whether, whetstone, whew, which, whichever, while, whine (-ed, -ing), whiner, whip (n.), whip (-ed, -ing), whirl (-ed, -ing), whiskbroom, whiskers, whisper (n.), whisper (-ed, -ing), whistle (n.), whistle (-ed, -ing), whistling (n.), white (-er, -est), whitewash (n.), whitewash (-ed, -ing), whittle (-ed, -ing), who, whoa, whoever, whole, whoop (-ed, -ing), whooping-cough, whopper, whose, why, wick, wicked, wide (-er, -est), wife, wiggle (ed, -ing), wiggler, wild, will, willing, wind (n.), wind (-ing), windmill, window, windy (-er), wine, wing, wink (-ed, -ing), winter, wintery, wipe (-ed, -ing), wiping-towel, wire, Wisconsin, wish (n.), wish (-ed, -ing), wishbone, with, withered, without, wolf, woman, women, wonder (-ed, -ing), wonderful, won't, wood, woodshed, woodpile, woods, wool, woolly, word, wore, work (n.), work (-ed, -ing), worker, world, 
worm, wormhole, wormy, worry, worse, worst, worth, would, wouldn't, wound, "wow," wrap (-ed, -ing), wrapper, wraps, wreck, wrestle (n.), wrestle (-ed, -ing), wring (-ing), wringer, wrinkle (n.), wrinkle (-ed, -ing), wrinkly, wrist, write (-ing), writer, writing (n.), written, wrong (a.), wrong (adv.), " wrongwards," wrote, wrung.

Yard, yarn, year, yeast, yell (n.), yell (-ed, -ing), yellow, yes, Yes

Mam, yesterday, yet, yolk, young (-er, -est), you, your, yours, yourself. Zinc.

Note.-Names of 105 persons and 15 animals are omitted. 